\title{
OBJECT INDEX
}

For classical novae the name is followed in brackets by ' $N$ ' and the year of outburst. The following symbols are used for the other objects: $R N=$ recurrent nova, $D N=$ dwarf nova, $\mathrm{NL}=$ nova-like, $\mathrm{XN}=\mathrm{X}$-ray nova, $\mathrm{IP}=$ intermediate polar, $\mathrm{DS}=$ detached system, $\mathrm{SN}$ $=$ supernova, $\mathrm{SYN}=$ symbiotic nova, $\mathrm{SYS}=$ symbiotic star, $\mathrm{mm}=$ missing star. Page numbers follwed by ' + ' are those of the title page of the article in which the object is mentioned several times, or it is the main topic of the article. Page numbers followed by ' $d$ ' refer to displayed material (tables or figures).

Z And (SYS) $442 d$

EG And (SYS) 442d

OS And (N1986) 115+, 117d, 118d, 119d, 123d, 142d, 144, 210+, 211d, 212d

RX And (DN) 346, 348

AE Aqr (IP) $3+, 74 \mathrm{~d}$

FO Aqr (IP) $31 \mathrm{~d}, 50+, 51 \mathrm{~d}, 74 \mathrm{~d}$

DO Aql (N1925) 38, 86

V356 Aql (N1936) 15d, 82

V500 Aql (N1943) 180

V603 Aql (N1918) 7, 13+, 15d, 18d, 34, 65, 73+, 73d, 74d, 79+, 98d, 101d, 155, 180, 185, 348,398

V605 Aql (N1919) 40, 95, 180

V794 Aql (NL) 31d

V1229 Aql (N1970) 15d, 142d, 180, 345, 348

V1301 Aql (N1975) 142, 142d

V1315 Aql (NL) 31d, 39

V1370 Aql (N1982) 97+, 98d, 104d, 115+, 119d, 120d, 124d, 132, 133, 140, 142d, 169, 171, $188+, 191 \mathrm{~d}, 198,205 \mathrm{~d}, 215+, 220 \mathrm{~d}, 250,253,254,359 \mathrm{~d}, 373+, 375 \mathrm{~d}, 376 \mathrm{~d}$

AE Ara (SYS) $445 \mathrm{~d}$

OY Ara (N1910) 15d

W Ari (mm, N1855) 34

$\underline{\text { SU Ari }}$ (mm, N1854) 34

TT Ari (NL) 31d, 346

T Aur (N1891) 7, 20d, 25d, 28, 65, 66d, 67, 74d, 98d, 103d, 180, 185, 192, 205d, 220d, $293,342+, 359 \mathrm{~d}, 375 \mathrm{~d}, 376 \mathrm{~d}, 377 \mathrm{~d}$

KR Aur (NL) $31 \mathrm{~d}$

UV Aur (SYS) 442d, 443d

V363 Aur (NL) 31d

T Boo (N1860) 38

$\underline{\mathrm{Z} \text { Cam }}$ (DN) 7, 232

$\underline{\mathrm{AC} \mathrm{Cnc}}(\mathrm{NL}) 31 \mathrm{~d}$

$\underline{\mathrm{YZ} \mathrm{Cnc}}$ (DN) 231, 232, 232d, 451

BG CMi (IP) 31d, 74d

$\eta$ Car 180 
QU Car (NL) 31d

B Cas (SN) 34

V425 Cas (NL) 31d

V592 Cas (NL) 31d

BV Cen (DN) $180,345,347 \mathrm{~d}$

V842 Cen (N1988) 92, 93d, 138+, 142d, 143d, 197+, 197d, 206+, 259-261, 400, 431+, 432d

IV Cep (N1971) 13+, 15d, 49d, 98d, 101d, 180, 345, 348

Z Cha (DN) 64

RR Cha (N1953) 38

AR Cir (N1906) 30

TV Col (IP) 31d, 74, 74d

V394 CrA (RN) 134, 348, 405+, 425, 425d, 426d

V655 CrA (N1967) 15d

V693 CrA (N1981) 38, 97+, 98d, 104d, 115+, 117d, 118d, 119d, 132, 188+, 191d, 205d, 220d, 221, 222, 348, 359d, 373+, 375d, 376d, 377d

T CrB (RN) 3+, 180, 405+, 411d, 425d, 426d

Q Cyg (N1876) 13+, 15d, 20d, 48+, 49d, 66d, 180, 345, 347d

BF Cyg (SYS) $442 \mathrm{~d}$

CH Cyg (SYS) 418, 442d

CI Cyg (SYS) 442d

EM Cyg (DN) 452, 453

SS Cyg (DN) 5, 7, 346

V404 Cyg (XN) 408, 425, 429+, 430

V407 Cyg (SYS) 440d, 442d

V450 Cyg (N1942) 180

BV465 Cyg (N1948) 15d

V476 Cyg (N1920) 34, 180, 185

V751 Cyg (NL) 31d

V1016 Cyg (SYN) 349, 416+, 416d, 422d, 437, 440d, 442d

V1329 Cyg (SYN, HBV 475) 416+, 416d, 422d, 431+, 431d, 437, 440d, 442d

V1500 Cyg (N1975) 14, 15d, 21, 24+, 34, 45, 47, 59+, 60d, 63, 64, 68+, 69d, 70d, 74d,

$79+, 84 \mathrm{~d}, 87 \mathrm{~d}, 97+, 98 \mathrm{~d}, 100 \mathrm{~d}, 127+, 138+, 139 \mathrm{~d}, 142 \mathrm{~d}, 148,150,161+, 161 \mathrm{~d}, 162 \mathrm{~d}$, $169+, 171 \mathrm{~d}, 174 \mathrm{~d}, 177 \mathrm{~d}, 180,188+, 191 \mathrm{~d}, 195+, 195 \mathrm{~d}, 205 \mathrm{~d}, 215+, 220 \mathrm{~d}, 256,303,303 \mathrm{~d}$, $321,342+, 356,359 \mathrm{~d}, 373+, 375 \mathrm{~d}, 376 \mathrm{~d}, 377 \mathrm{~d}, 399$

V1668 Cyg (N1978) 25d, 74d, 87d, 98d, 104d, 115+, 117d, 118d, 119d, 123d, 142d, 157, $188+, 191 \mathrm{~d}, 205 \mathrm{~d}, 216,217,220 \mathrm{~d}, 249-250,356,359 \mathrm{~d}, 374,375 \mathrm{~d}, 376 \mathrm{~d}, 377 \mathrm{~d}$

V1819 Cyg (N1986) 142d, 144, 169, 171, 210+, 211d, 376d

CM Del (NL) 31d

HR Del (N1967) 15d, 25d, 30, 65, 74d, 79+, 81d, 85d, 87d, 97+, 98d, 102d, 107d, 109d, $169+, 174 \mathrm{~d}, 179+, 192,205 \mathrm{~d}, 245,319,342+, 359 \mathrm{~d}, 375 \mathrm{~d}, 376 \mathrm{~d}, 377 \mathrm{~d}$

AA Dor (DS) 453

4 Dra 452

U Gem (DN) 7, 59,60d, 64

DN Gem (N1912) 15d, 65, 180 
V Per (N1887) 24t, 74d

AX Per (SYS) $442 \mathrm{~d}$

GK Per (N1910) 7, 13+, 15d, 16d, 19d, 20d, 25d, 34, 65, 74d, 79+, 94d, 98d, 111d, 155, $156 \mathrm{~d}, 169+, 179+, 208,216,251,342+, 347 \mathrm{~d}$

SZ Per (mm, N1853) 34

V741 Per (SYS) 442d, 443

RR Pic (N1925) 13+, 15d, 20d, 65, 74d, 79+, 98d, 100d, 111, 130, 180, 185, 192, 205d, $208+, 209 \mathrm{~d}, 220 \mathrm{~d}, 342+, 359 \mathrm{~d}, 375 \mathrm{~d}, 376 \mathrm{~d}, 377 \mathrm{~d}$

AO Psc (IP) 31d, 74d

AS PSC (DN) 408

CP Pup (N1942) 13+, 15d, 16d, 24+, 34, 45, 61+, 62d, 63+, 64d, 65+, 66d, 67, 74d, 79+, 93d, 98d, 101d, 180, 190, 220d, 293, 333, 345, 348

HZ Pup (N1963) 15d

RX Pup (SYS) 445d

VV Pup (DN) 7,346

T Pyx (RN) 24, 180, 220d, 348, 405+, 425d, 426d, 427+

HM Sge (SYN) 349, 416+, 416d, 422d, 433+, 433d, 434d, 435+, 436d, 437+, 438d, 439d, 440d, 442d, 443d

WY Sge (N1783) 18d, 31, 34, 39, 74d, 80, 180,320, 345, 347d

WZ Sge (N1913) 7-8, 59+, 60d

GR Sgr (N1924) 15d

HS Sgr (N1900) 30

V441 Sgr (N1930) 15d

V999 Sgr (N1910) 15d

V1016 Sgr (N1899) 15d, 30

V1017 Sgr (RN) 345, 347d, 425d, 426d

V1223 Sgr (IP) 31d, 74d

V2572 Sgr (N1969) 15d

V3885 Sgr (NL) 31d, 280, 280d, 285d

V3890 Sgr (N1962) 13, 345, 346, 347d

V4021 Sgr (N1977) 142d

V4077 Sgr (N1982) 348

V4135 Sgr (N1987) 142d

T Sco (N1860) 38, 43

U Sco (RN) 134, 220d, 236+, 241d, 348, 405+, 425, 425d, 426d, 428

CL Sco (NL) 31d

V720 Sco (N1950) 42

V745 Sco (RN) 425+, 42d, 426d, 444+, 445d

VY Scl (NL) 31d

$\mathrm{VZ} \mathrm{Scl}$ (NL) 346

N Sct $198987 \mathrm{~d}$

EU Sct (N1949) 15d

FS Sct (N1952) 15d, 30

V368 Sct (N1970) 15d, 180 
SY Gem $(\mathrm{mm}) 34$

$\mathrm{VZ} \mathrm{Gem}(\mathrm{mm}) 34$

AM Her (NL) 60, 346, 451

DQ Her (N1934) 6, 9, 15d, 20d, 24+, 25d, 34, 65, 74d, 82, 97, 98d, 102d, 179+, 190, 192, 205d, 208, 253, 208, 253, 291, 293 +, 294d, 299, 313, 333, 342, 359d, 367, 374, 375d, 276d, $377 \mathrm{~d}, 411 \mathrm{~d}, 451$

HZ Her 74

V446 Her (N1960) 13+, 15d, 20d, 49d, 180, 345, 346, 347d, 376d

V533 Her (N1963) 15d, 25d, 66d, 67, 74d, 80, 98d, 103d, 180, 376d

V795 Her (IP) 24, 31d, 74d

V825 Her (NL) 31d

V827 Her (N1987) 141, 142d, 144, 169, 210+, 211d, 212d

EX Hya (IP) 26, 74d

VW Hyi (DN) $59,60 \mathrm{~d}$

CP Lac (N1936) 15d, 34, 98d, 101d, 180, 185, 359d, 376d

DI Lac (N1910) 15d, 16, 21, 25d, 66d, 67, 74d, 180, 348

DK Lac (N1950) 65, 98d, 104d, 155, 156d, 180, 359d, 376d

U Leo (N1855) 19, 34

$\underline{\mathrm{RX} \mathrm{LMi}}$ (NL) 31d

$\beta$ Lyr 5

HR Lyr (N1919) 65, 180

MV Lyr (NL, McRae 43.1) 6, 31d, 346

BT Mon (N1939) 15d, 25d, 28, 65, 66d, 67, 74d, 180, 346

GI Mon (N1918) 15d

KQ Mon (NL) 31d

V616 Mon (XN) 425, 453

GQMus (N1983) 24+, 74d, 92, 98d, 103d, 115+, 119d, 120d, 121d, 124d, 127+, 142d, $148+, 149 \mathrm{~d}, 154 \mathrm{~d}, 188+, 191 \mathrm{~d}, 202+, 202 \mathrm{~d}, 203 \mathrm{~d}, 205 \mathrm{~d}, 215+, 220 \mathrm{~d}, 250,299,310,348$, $374,375 \mathrm{~d}, 376 \mathrm{~d}, 377 \mathrm{~d}, 450$

N Oph 193838,43

RS Oph (RN) 97, 115, 134, 150, 169, 170, 180, 190, 405+,411d, 418, 423+, 424d, 427+

V380 Oph (NL) 31d

V426 Oph (IP) $31 \mathrm{~d}, 74 \mathrm{~d}$

V442 Oph (NL) 31d, 326

V840 Oph (N1917) 34

V841 Oph (N1848) 13+, 15d, 16d, 18d, 20d, 34, 48+, 49d, 61+, 62d, 66d, 67, 180

V2051 Oph (DN) 27

V2110 Oph (SYN, AS 239) 416+, 416d, 440d

V2214 Oph (N1988) 142d, 200+, 201d

GR Ori (N1916) 38

AR Pav (SYS) 445d

AG Peg (SYN) 349, 416+, 416d, 422d, 432, 440d, 442d

RU Peg (DN) 7, 346, 348

RX Peg (DN) 7 
V373 Sct (N1975) 180

X Ser (N1903) 38, 65, 165+, 165d, 166d

FH Ser (N1970) 15d, 79+, 94d, 115, 142d, 169+, 171d, 174d, 180, 248-249, 248d, 251, 253, $295,345,348$

LX Ser (NL) 31d

LW Ser (N1978) 142d

RT Ser (RN) 38, 349, 358, 416+, 416d, 422d, 440d

RX Sex (NL) 31d, 229d, 280, 280d, 281d, 282d

SW Sex (NL) 228

$\underline{\mathrm{Y} T a u} 198$

OV Tau 34

XX Tau (N1927) 180

V471 Tau (DS) 34, 42, 452

RR Tel (SYN) 19, 98d, 103d, 349, 358, 376d, 416+, 416d, 422d, 432, 437, 440d

RW Tri (NL) 7, 31d, 230d, 326

NSV00856 Tri (mm, N1853) 34

AN UMa (DN) 346

DW UMa (NL) 31d

SU UMa (DN) 231, 231d, 232, 451

SW UMa (IP) 26, 74d

UX UMa (NL) 6, 7, 31d, 230d, 326

RW UMi (N1956) 34, 38, 180, 348

IX Vel (NL) 31d, 277+, 278d, 280d, 284d

HS Yir (NL) $31 \mathrm{~d}$

N Vul $1989345,346,347 \mathrm{~d}$

CK Vul (N1670) 9, 13+, 18d, 31, 34+, 79+, 180, 320, 345

LV Vul (N1968) 15d, 98d, 104d, 163+, 163d, 180

NQ Vul (N1976) 88, 139d, 142d, 199, 253, 257

PU Vul (SYN) 416+, 416d, 422d, 440d, 442d

PW Vul (N1984) 115+, 117d, 123d, 134, 142d, 148+, 149d, 159+, 160d, 169, 171, 189, 191d, 204+, 205d, 210+, 211d, 224, 258, 348, 375d, 376d, 377d

QU Vul (N1984) 115+, 119d, 127+, 138+, 142d, 148+, 149d, 169+, 175d, 192, 221, 224

QV Vul (N1987) 133, 136, 138+, 142d, 144d

\section{$\underline{126 \mathrm{AD}} 34$}

$\underline{396 \mathrm{AD}} 34,42$

$\underline{839 \mathrm{AD}} 34$

$891 \mathrm{AD} 34$

$1437 \mathrm{AD} 34,57+, 58 \mathrm{~d}$

N1926 in LMC 72

N1948 in LMC 72

N1988-1 in LMC 115+, 119d, 123d, 125d, 141, 142d, 157+, 272, 274d

N1988-2 in LMC 115+, 119d, 125d, 142d, 157+

$\mathrm{N} 6$ in M31 45d 
N 26 in M31 $45 \mathrm{~d}$

N 29 in M31 45d

N 31 in M31 45d

SN 1987a in LMC 129, 181

AS 239 see V2110 Oph

$\underline{\mathrm{AS} 296}$ (SYS) 440+, 441d

AS 338 (SYS) $440 \mathrm{~d}$

AS 360 (SYS) 442d

H0542-407 (IP) 74d

He2-38 (SYS) 445d

He2-407 (SYS) 442d

He2-468 (SYS) 442d

IRAS 06518-1041 55+

LB1800 (NL) 31d

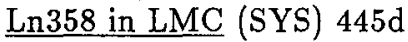

PG2133+115 (NL) 31d

PHL227 283, 286d

Sanduleak star in LMC (SYS) 445d

SS 43374

1H0709-360 (IP) 24, 26

$\underline{0623+71}$ (NL) 180 